LA-UR-99-3356

Approved for public release;

distribution is unlimited.

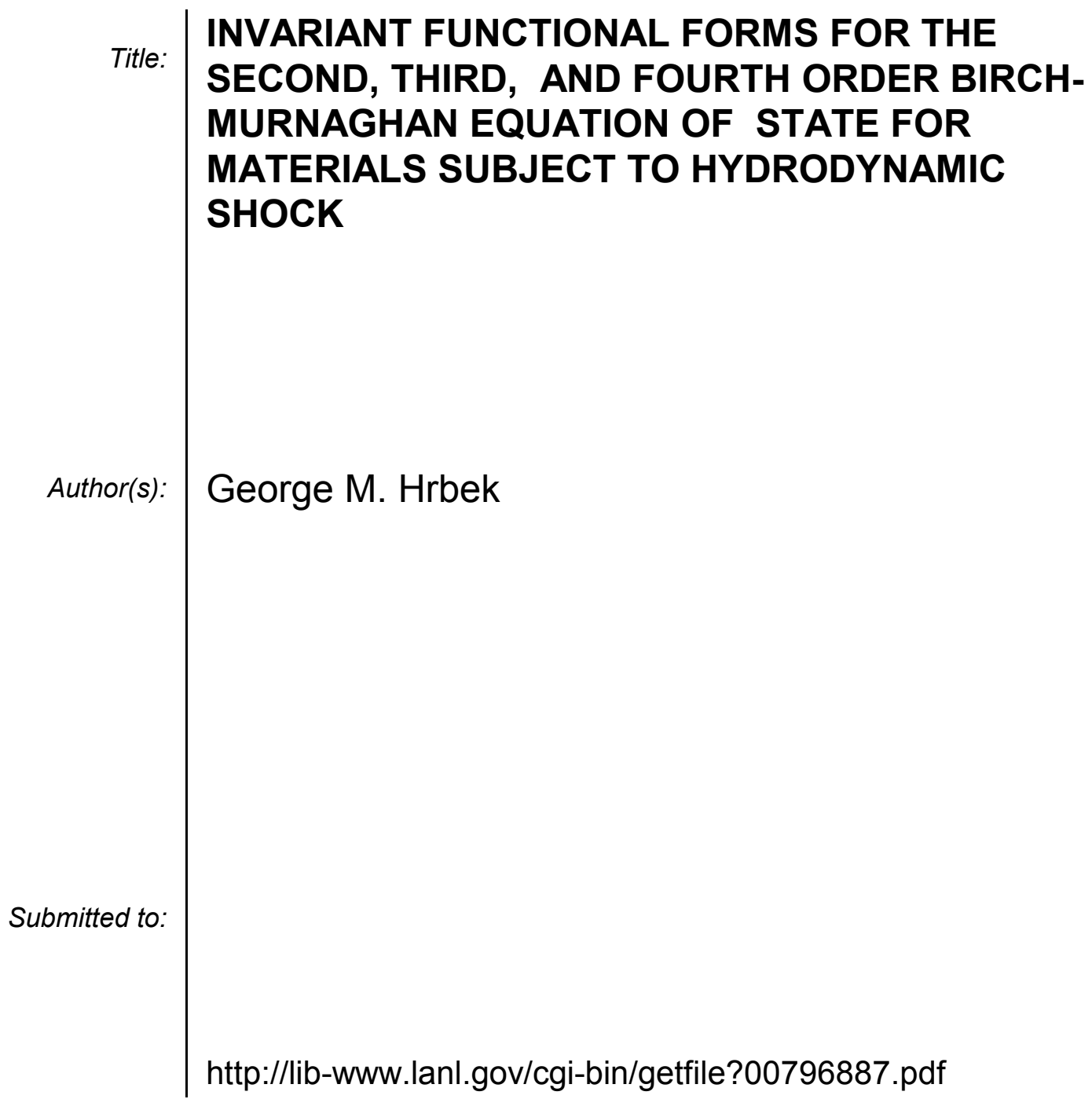

Los Alamos National Laboratory, an affirmative action/equal opportunity employer, is operated by the University of California for the U.S. Department of Energy under contract W-7405-ENG-36. By acceptance of this article, the publisher recognizes that the U.S. Government retains a nonexclusive, royaltyfree license to publish or reproduce the published form of this contribution, or to allow others to do so, for U.S. Government purposes. Los Alamos National Laboratory requests that the publisher identify this article as work performed under the auspices of the U.S. Department of Energy. Los Alamos National Laboratory strongly supports academic freedom and a researcher's right to publish; as an institution, however, the Laboratory does not endorse the viewpoint of a publication or guarantee its technical correctness. 


\title{
INVARIANT FUNCTIONAL FORMS FOR THE SECOND, THIRD, AND FOURTH ORDER BIRCH-MURNAGHAN EQUATION OF STATE FOR MATERIALS SUBJECT TO HYDRODYNAMIC SHOCK
}

\author{
George M. Hrbek
}

Los Alamos National Laboratory, Los Alamos, NM 87545

\begin{abstract}
Group Theoretical Methods as defined by Lie are applied to the problem of temperature independent, hydrodynamic shock in a Birch-Murnaghan continuum. Ratios of the group parameters are shown to be linked to physical parameters specified in the second, third, and fourth order BM-EOS approximations. We provide an illustrative example of how the expansion ratio $\left(\mathrm{c}_{1} / \mathrm{c}_{2}\right)$ and the material resistance condition $\left(\mathrm{c}_{4} / \mathrm{c}_{2}\right)$ are a function of the compression ratio $\beta$, for a spherical shock wave propagating through $\mathrm{MgO}$ and $\mathrm{Na}$.
\end{abstract}

\section{INTRODUCTION}

The use of group invariance techniques in the solution of the hydrodynamic flow problem is well known. ${ }^{(1)}$ Traditional application of these methods has centered on reducing the order for these coupled sets of linear, hyperbolic PDE's by coordinate transformations.

Since the hydrodynamic flow equations are characteristically hyperbolic, the reduction to nonlinear ODE's offers a great advantage to the numerical solution of these types of problems.

The most widely used coordinate transformation in the literature is the self-similar group. This reduction assumes a time scaling of the flow variables (i.e., density, pressure, and velocity) wherein the modified dimensionless profiles retain a constant shape scaled only in time.

These solutions and the problems they represent are the conditions of a physically degenerate and asymptotic state. Problems of this type exhibit self-similarity of the first kind.

Typically, one could apply this technique to simulate the physically degenerate problem of a strong shock (i.e., blast wave) with no counter pressure $\mathrm{c}_{4}$, and high Mach number flow. ${ }^{(2)}$

\section{THEORY}

We will now apply the Lie algorithm to the 1-D hydrodynamic flow equations of continuity,

$\frac{\partial}{\partial t}\left(\rho r^{j}\right)+\frac{\partial}{\partial r}\left(u \rho r^{j}\right)=0$

And the conservation of linear momentum,

$\frac{\partial}{\partial t}\left(u \rho r^{j}\right)+\frac{\partial}{\partial r}\left(\left(u^{2}+\frac{P}{\rho}\right) u \rho r^{j}\right)=0$.

And the conservation of energy,

$$
\begin{aligned}
& \frac{\partial}{\partial t}\left(\left(\varepsilon+\frac{u^{2}}{2}\right) \rho r^{j}\right)+ \\
& +\frac{\partial}{\partial r}\left(\left(\varepsilon+\frac{u^{2}}{2}+\frac{P}{\rho}\right) u \rho r^{j}\right)=0 .
\end{aligned}
$$

Where $\mathrm{j}=0,1$, or 2 for rectangular, cylindrical, and spherical geometry respectively.

These equations require a statement of closure that represents the equation of state. In this presentation we will consider a one-dimensional flow 
field wherein the equation of state is expressed as a bulk modulus $\mathrm{K}$, in density and pressure. It is to the admissible forms of $\mathrm{K}$ for the finite strain Birch-Murnaghan EOS's and how they relate to the group parameters that the rest of this paper will be devoted.

\section{GROUP STRUCTURE}

Application of the group analysis technique will be demonstrated for the spherical shock wave problem (i.e., $\mathrm{j}=2$ ). The flow equations for this problem are as follows;

$\rho_{t}+\rho u_{r}+u \rho_{r}+j u \rho r^{-1}=0$,

$u_{t}+u u_{r}+u \rho_{r}+\rho^{-1} P_{r}=0$,

$P_{t}+u P_{r}+K(\rho, P)\left\{u_{r}+j u r^{-1}\right\}=0$.

Use of the Lie algorithm leads to the following five-parameter group generator; ${ }^{(2)}$

$$
\begin{aligned}
\hat{U}= & c_{1} r \partial_{r}+\left(c_{2} t+c_{3}\right) \partial_{t}+c_{5} \rho \partial_{\rho}+\left(c_{1}-c_{2}\right) u \partial_{u} \\
& +\left(\left(c_{5}+2\left(c_{1}-c_{2}\right)\right) P+c_{4}\right) \partial_{P}
\end{aligned}
$$

This problem is further restricted by the socalled invariance condition on the mathematical structure of the bulk modulus $\mathrm{K}$.

$$
\begin{aligned}
& c_{5} \rho \frac{\partial K}{\partial \rho}+\left[\left(c_{5}+2\left(c_{1}-c_{2}\right)\right) P+c_{4}\right] \frac{\partial K}{\partial P}= \\
& =\left(c_{5}+2\left(c_{1}-c_{2}\right)\right) K
\end{aligned}
$$

From the group generator a characteristic set of equations can be written that can be used to generate various coordinate transformations for the governing set of flow equations

$$
\begin{aligned}
\frac{d r}{c_{1} r} & =\frac{d t}{c_{2} t+c_{3}}=\frac{d \rho}{c_{5} \rho}= \\
& =\frac{d u}{\left(c_{1}-c_{2}\right) u}=\frac{d P}{\left\{c_{5}+2\left(c_{1}-c_{2}\right)\right\} P+c_{4}}
\end{aligned}
$$

Given this set of equations, it is then possible to construct the following general class of similarity variables;

$$
\lambda=r\left(t+c_{3} / c_{2}\right)^{-c_{1} / c_{2}}
$$

$$
\rho(\lambda, t)=\left(t+c_{3} / c_{2}\right)^{c_{5} / c_{2}} F(\lambda),
$$

$$
u(\lambda, t)=\left(t+c_{3} / c_{2}\right)^{c_{1} / c_{2}-1} G(\lambda),
$$

and

$$
\begin{aligned}
P(\lambda, t) & =\left(\frac{c_{5}}{c_{2}}+2\left(\frac{c_{1}}{c_{2}}-1\right)\right)^{-1} \times \\
\times & {\left[\left(t+\frac{c_{3}}{c_{2}}\right)^{\left(\frac{c_{5}}{c_{2}}+\frac{2 c_{1}}{c_{2}}-2\right)} H(\lambda)-\frac{c_{4}}{c_{2}}\right] }
\end{aligned}
$$

Note that these new variables are expressed in terms of the independent variable $\lambda$, the time $t$, the group parameters $\mathrm{c}_{1}$ through $\mathrm{c}_{5}$, and the reduced functions, $\mathrm{F}(\lambda), \mathrm{G}(\lambda)$, and $\mathrm{H}(\lambda)$.

\section{BIRCH-MURNAGHAN EOS's}

One application of the group approach is to provide a technique for comparing a theoretical equation of state with experimental data.

To illustrate this point, the invariance conditions for the second, third, and fourth order BirchMurnaghan Equations of State will be determined.

Consider the fourth order Birch-Murnaghan pressure and bulk modulus. $^{(3)}$

$$
P_{4 t h}=3 f(1+2 f)^{5 / 2} K_{O T}\left[1-2 \xi f+4 \zeta f^{2}\right],
$$

$$
\begin{aligned}
& \text { and } \\
& \begin{aligned}
K_{4 t h} & =5 f(1+2 f)^{5 / 2} K_{O T}\left[1-2 \xi f+4 \zeta f^{2}\right]+ \\
& +(1+2 f)^{7 / 2} K_{O T}\left[1-4 \xi f+12 \zeta f^{2}\right]
\end{aligned}
\end{aligned}
$$

Where, 
$f=\left(\beta^{2 / 3}-1\right) / 2$

$\xi=3\left(4-K_{O T}^{\prime}\right) / 4$

and

$\left.\zeta=3\left(K_{O T} K_{O T}^{\prime \prime}+K_{O T}^{\prime} \mid K_{O T}^{\prime}-7\right\rfloor+143 / 9\right) / 8$.

Note that $\mathrm{K}_{\mathrm{OT}}, \mathrm{K}_{\mathrm{OT}}$ ', and $\mathrm{K}_{\mathrm{OT}}$ ', are the isothermal bulk modulus at ambient temperature, and its first and second derivatives respectively.

\section{INVARIANCE CONDITION FOR THE BULK MODULUS}

The invariance condition on the bulk modulus requires that the mathematical form be as follows;

$K=\kappa(f) P$.

So that,

$\kappa(f)=\frac{5}{3}+\frac{(1+2 f)\left[1-4 \xi f+12 \zeta f^{2}\right]}{3 f\left(1-2 \xi f+4 \zeta f^{2}\right)}$.

This can then be substituted into the invariance condition on the bulk modulus function to obtain the ratio $\mathrm{c}_{1} / \mathrm{c}_{2}$

\section{INVARIANCE OF THE FIRST AND SECOND KIND}

Classically, the self-similar blast wave problem considers an asymptotic solution (self-similarity of the first kind). ${ }^{(1)}$ This requirement manifests itself in the ratios $\left(c_{1} / c_{2}\right)$ and $\left(c_{5} / c_{2}\right)$ possessing single values, applicable for a particular material for degenerate problems only.

As an example, consider an ideal gas and a blast wave propagating through it. In this case, $\mathrm{c}_{1} / \mathrm{c}_{2}$ is equal to $2 / 5$, $\left(\mathrm{c}_{5}=0\right.$, i.e., for a uniform media).

In this study a more general class of problems is suggested by inspection of the ratio $\left(c_{1} / c_{2}\right)$. This situation was created because all of the group parameters expressed in the invariance condition for the mathematical form $\mathrm{K}(\rho, \mathrm{P})$ were maintained to affect as general solution as possible. This leads us to a statement of self-similarity of the second kind.

These represent physical problems for conditions where a finite (non-zero) limit on the group parameters do not exist over all parameter space considered in the physical problem (i.e., it varies), but which approach the classical self-similar problem as a special case.

\section{ILLUSTRATIVE EXAMPLES}

To illustrate how this technique can be applied to study real physical problems, we will consider

$\frac{c_{1}}{c_{2}}=\frac{2}{5}+\frac{c_{4}(1+2 f)^{5 / 2}}{5 c_{2} \beta^{4} K_{O T}}\left\{\frac{5 f\left(1-2 \xi f+4 \zeta f^{2}\right)+(1+2 f)\left(1-4 \xi f+12 \zeta f^{2}\right)}{\left(1-4 \xi f+4 \xi(1+2 \xi) f^{2}-32(1+\xi) \zeta f^{3}+16 \zeta(1+3 \zeta) f^{4}\right)}\right\}$

Reduction to third and second order forms is accomplished by setting $\zeta=0$, and then $\zeta=0$ and $\xi=0$ respectively.

These conditions express mathematically how the expansion ratio $\left(\mathrm{c}_{1} / \mathrm{c}_{2}\right)$ and the material resistance condition $\left(\mathrm{c}_{4} / \mathrm{c}_{2}\right)$ are related and vary with the compression ratio $\beta$.

Note that the material uniformity ratio $\left(c_{5} / c_{2}\right)$ is related to $\left(c_{1} / c_{2}\right)$ by requiring constant energy in the shock wave, as expressed in the requirement that the energy integral remain invariant. This condition is satisfied when $\mathrm{c}_{5}=2 \mathrm{c}_{2}-5 \mathrm{c}_{1}$.
Sodium $(\mathrm{Na})$ and Magnesium Oxide $(\mathrm{MgO}) .{ }^{(4)}$ These are solids that exhibit quite different expansion profiles $\left(\mathrm{c}_{1} / \mathrm{c}_{2}\right)$.

Figures 1 through 6 show the variation in the expansion ratio $\left(\mathrm{c}_{1} / \mathrm{c}_{2}\right)$ as a function of the material resistance condition $\left(\mathrm{c}_{4} / \mathrm{c}_{2}\right)$ for differing degrees of approximation in the Birch-Murnaghan EOS.

Magnesium Oxide $\left(\mathrm{K}_{\mathrm{OT}}=160.3 \mathrm{GPa}, \mathrm{K}_{\mathrm{OT}}{ }^{\prime}=4.1\right.$, $\mathrm{K}_{\mathrm{OT}}{ }^{\prime \prime}=-0.03 \mathrm{GPa}^{-1}$ ) has a sharp drop in the expansion ratio $\left(c_{1} / c_{2}\right)$ with both increasing material resistance and the degree of approximation in the EOS for an increasing compression ratio $\beta$.

Application of the coefficients for Sodium $(\mathrm{Na})$ $\left(\mathrm{K}_{\mathrm{OT}}=6.028 \mathrm{GPa}, \mathrm{K}_{\mathrm{OT}}{ }^{\prime}=4.27, \mathrm{~K}_{\mathrm{OT}}{ }^{\prime \prime}=-0.4 \mathrm{GPa}^{-1}\right)$ yield quite different results. 
While the results for the second and third degree approximations possess similar trends as seen for $\mathrm{MgO}$, the fourth degree approximation develops a hump as the compression ratio rises (peaking at around $\beta=3.45$ ).

The reasons for this behavior will be explored in a future paper.

\section{REFERENCES}

(1) Barenblatt, G. I., Scaling, self-similarity, and intermediate asymptotics, Cambridge Univ. Press, 1996.

(2) Hrbek, G. M., Group Analysis of Shock Wave Phenomena in Solids, Ph.D. Dissertation, Univ. of IL, Urbana, IL, 1992.

(3) Jeanloz, R., Geophysical Research Letters 8(12) 1219-1222 (1981).

(4) Hofmeister, A. M., Journal of Geophysical Research 96 (B13), 21893-21907 (1991).

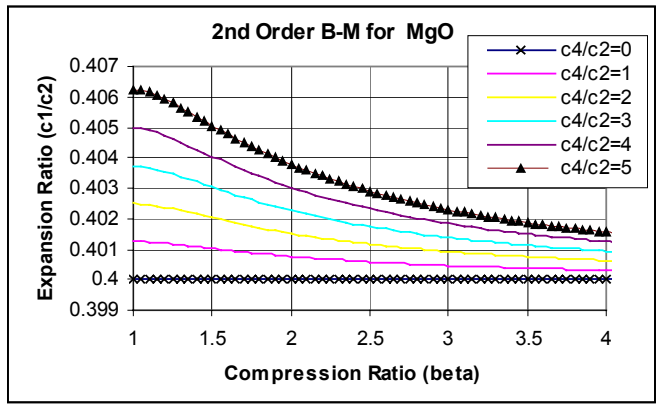

Figure 1

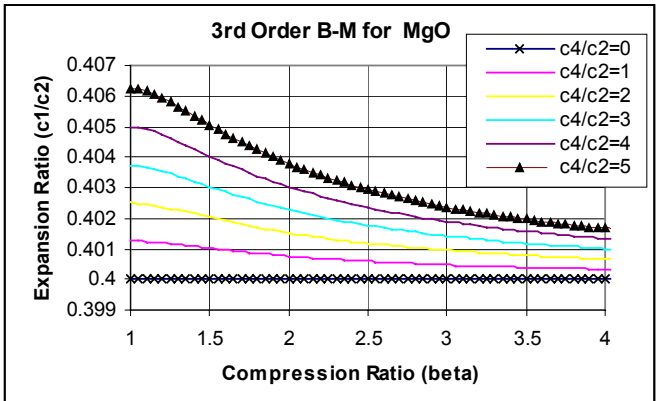

Figure 2

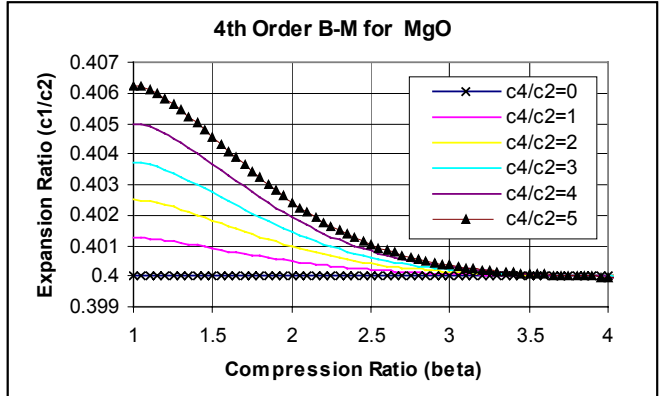

Figure 3

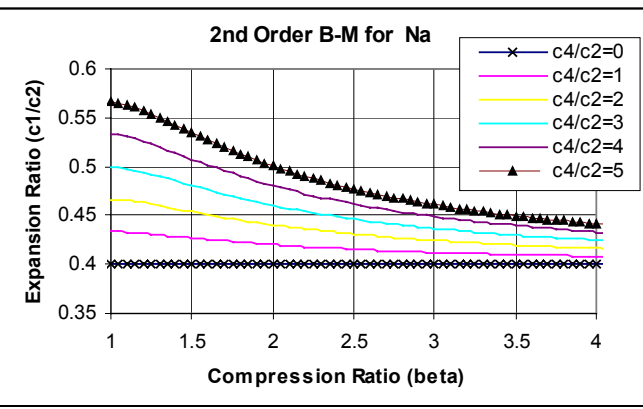

Figure 4

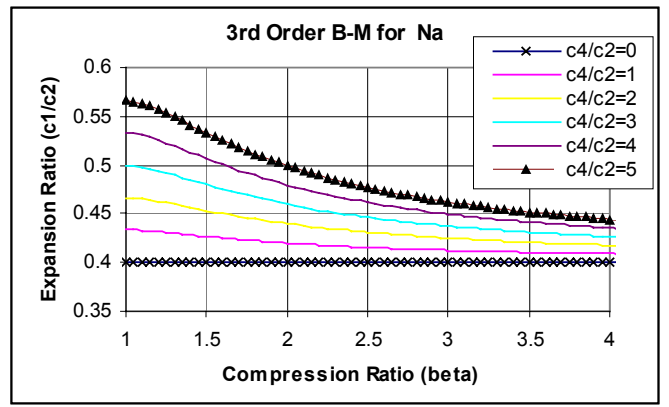

Figure 5

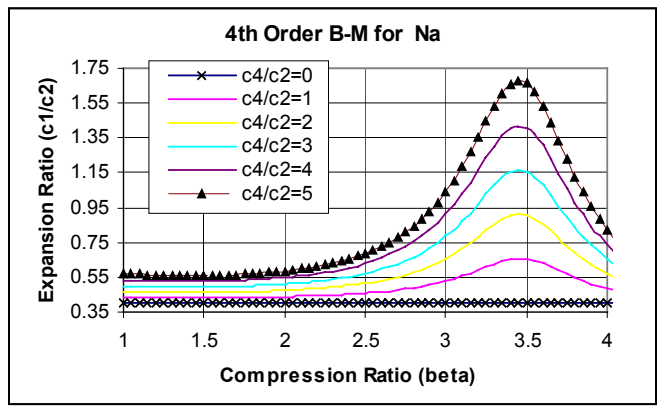

Figure 6 